\title{
AN IMPROVED CALIBRATION OF THE MIXING-LENGTH BASED ON SIMULATIONS OF SOLAR-TYPE CONVECTION
}

\author{
H.-G. LUDWIG \\ Astronomical Observatory, Niels Bohr Institute, \\ DK-2100 Copenhagen, Denmark, [hgl@astro.ku.dk]
}

B. FREYTAG

Institut für Astronomie und Astrophysik der Universität Kiel, D-24098 Kiel, F.R.G., [Freytag@astrophysik.uni-kiel.de]

\begin{abstract}
AND
M. STEFFEN

Astrophysikalisches Institut Potsdam, D-14473 Potsdam, F.R.G., [MSteffen@aip.de]
\end{abstract}

Based on detailed 2D numerical radiation hydrodynamics (RHD) calculations of timedependent compressible convection, we have studied the dynamics and thermal structure of the convective surface layers of stars in the range of effective temperatures and gravities between $4500 \mathrm{~K} \leq T_{\text {eff }} \leq 7100 \mathrm{~K}$ and $2.54 \leq \log g \leq 4.74$. Although our hydrodynamical models describe only the shallow, strongly superadiabatic layers at the top of the convective stellar envelope, they provide information about the value of the entropy $s^{*}$ of the deeper, adiabatically stratified regions. E.g. in the solar case the helioseismically measured entropy jump is predicted within $9 \%$ of its actual value.

Despite the complex interplay of hydrodynamics and radiative transfer we find a rather simple functional dependence $s^{*}\left(T_{\text {eff }}, \log g\right)$ across the HR diagram. $s^{*}$ can be translated into an effective mixing-length parameter $\alpha_{\text {MLT }}$ suitable for constructing standard stellar structure models by matching $s^{*}$ in envelope models basing on mixing-

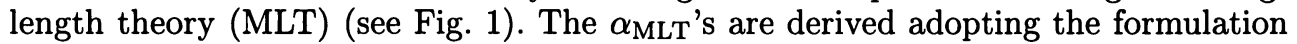
of MLT by Böhm-Vitense (Zs. Ap. 46, p.108, 1958). The atmospheric $T(\tau)$ relation in the envelope models was chosen to mimic closely the average structure of the RHD atmospheres in order to eliminate its influence on the calibration of $\alpha_{\mathrm{MLT}}$. We find a moderate, nevertheless significant variation of $\alpha_{\mathrm{MLT}}$ - primarily with effective temperature - over the range studied. A similar calibration of the convection theory by Canuto \& Mazzitelli (Ap.J. 370, p.295, 1991) extended by including a variable amount of overshoot does not lead to a smaller variation of the controlling parameter $\alpha_{\mathrm{CM}}$ (Fig. 2). The presented calibrations were derived for solar metallicity and helium content. Preliminary results of RHD models for lower metallicity show a complex dependence of $\alpha_{\mathrm{MLT}}$ on $T_{\text {eff }}$ and $\log g$. The MLT calibration is improved with respect to an earlier version (cf. Ludwig/Freytag/Steffen, in: Solar Convection and Oscillations, eds. F.P. Pijpers, J. Christensen-Dalsgaard, \& C. Rosenthal, in press). 


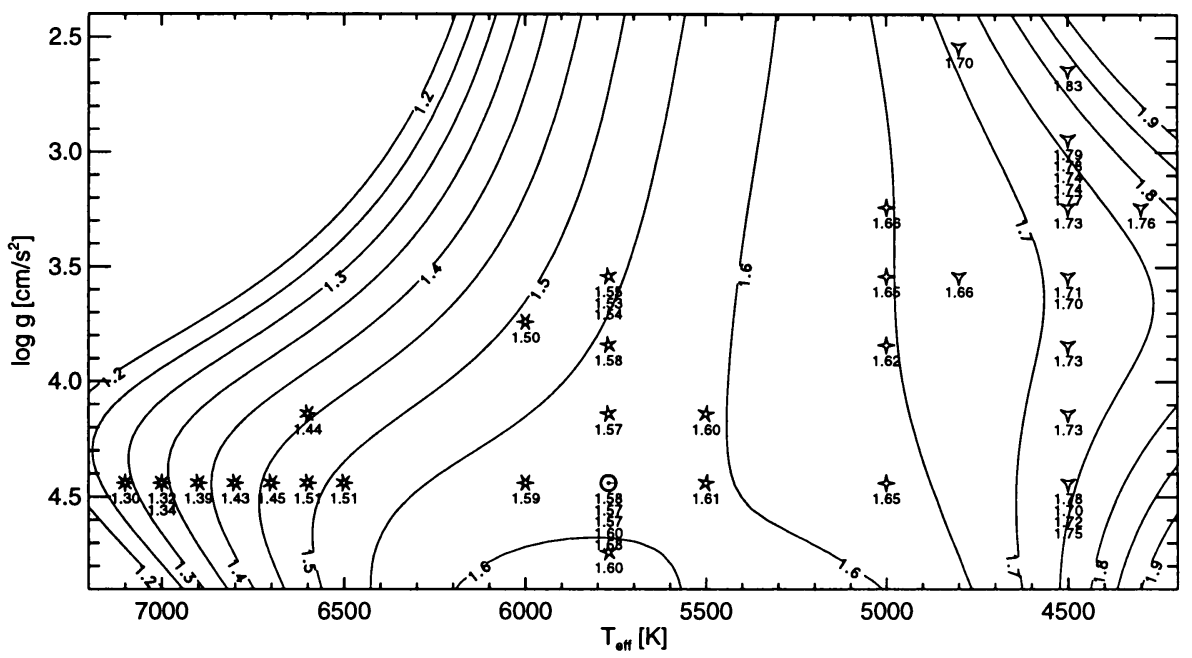

Figure 1. $\alpha_{\mathrm{MLT}}$ for standard mixing-length theory with $l_{\text {mix }}=\alpha_{\mathrm{MLT}} H_{\mathrm{p}}\left(l_{\text {mix }}\right.$ : mixing-length, $H_{\mathrm{p}}$ : local pressure scale height) derived from radiation-hydrodynamics (RHD) simulations as a function of effective temperature and gravitational acceleration. Symbols indicate RHD models. Attached to the symbols the actual data values are given, the contour lines present a polynomial fit to them. For some parameters several RHD models were computed. They differ in numerical details to provide an estimate of the internal uncertainty of the calibration.

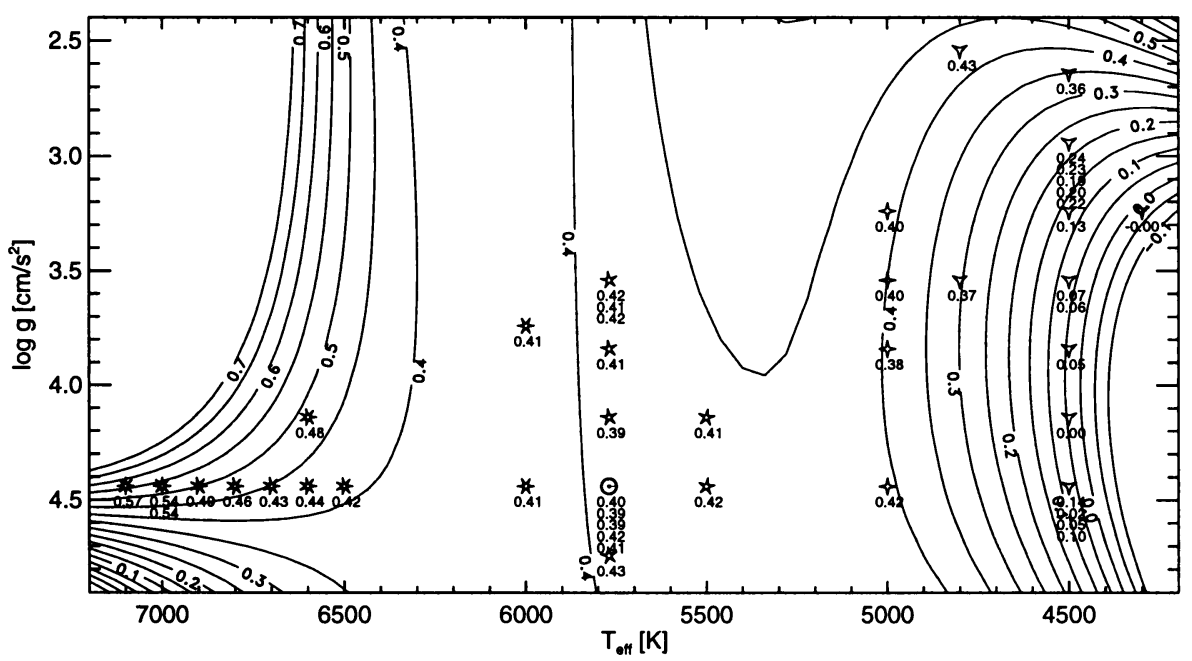

Figure 2. $\alpha_{\mathrm{CM}}$ for the Canuto \& Mazzitelli convection theory with $l_{\operatorname{mix}}=\Lambda+\alpha_{\mathrm{CM}} H_{\mathrm{p}, \mathrm{top}}$ ( $\Lambda$ : distance to the upper (Schwarzschild) boundary of the convective zone, $H_{\mathrm{p}, \text { top }}$ : pressure scale height at the upper boundary). The $\alpha_{\mathrm{CM}}$-calibration reproduces the same underlying $s^{*}$-distibution as the $\alpha_{\text {MLT }}$-calibration shown in Fig. 1. 